\title{
Behavioral Activation Treatment for Major Depressive Disorder: A Pilot Investigation
}

\author{
Jenifer M. Cullen, C. Richard Spates, Sherry Pagoto, Neal Doran
}

\begin{abstract}
Behavioral activation (BA) has emerged as an effective intervention for major depressive disorder. Previous research has indicated that it is as effective as the full cognitive behavioral treatment package (CBT). Conceptualized to consume fewer participant sessions, BA may be more efficient and cost-effective than CBT. With depression among the most common diagnoses in practice settings, NIMH's recent vision statement calls for continued research devoted to cost- and time-effective targeted treatment alternatives, including "behavioral activation as a more simply behavioral form of CBT" (NIMH, 2005, p. 92). The present investigation piloted a 10week individual intervention model of BA with medicated and unmedicated participants in a clinical research setting. This follows our earlier report (Porter, Spates, \& Smitham, 2004) of an efficacious group-administered BA intervention. In comparison to the group intervention, end state functioning of participants in the present investigation revealed more robust symptom reductions to within the fully recovered range of functioning. Keywords: depression, behavioral activation, behavior therapy, cognitive behavioral therapy.
\end{abstract}

Major depressive disorder (MDD) is the most prevalent psychological disorder (Kimerling, Ouimette, Cronkite, \& Moos, 1999) and is now the $4^{\text {th }}$ leading cause of disability across the lifespan and the $2^{\text {nd }}$ leading cause of disability among people aged 15-44 (World Health Organization, 2005). According to a National Comorbidity Survey Replication (NCS-R) the lifetime prevalence rate of MDD is $16 \%$ (Kessler et al., 2003). The prevalence of MDD is expected to rise such that by the year 2020, MDD will be second only to heart disease in terms of global burden of disease (World Health Organization, 2005). Although high, prevalence rates may be underestimated given that only about $70 \%$ of individuals with depression seek treatment (Angst, 1998). The effective treatment and prevention of MDD are of paramount importance and rank high among both mental health research and clinical objectives. A number of psychological interventions have been identified as holding at least partial efficacy towards this end. Among them are cognitive behavior therapy (CBT), cognitive therapy (CT), behavior therapy (BT), and interpersonal therapy (IT).

Both behavioral and cognitive theories of depression are supported with substantial clinical and empirical evidence (Beck, Rush, Shaw, \& Emery, 1979; Dobson, 1989; McLean, Ogston, \& Grauer, 1973; Steinbrueck, Maxwell, \& Howard, 1983), and distinct treatment modalities have emerged from each perspective. A behavioral conceptualization of depression first emerged in the 1970s, and suggested that depression is a result of a reduction in positively reinforcing behavior either via reduced availability of reinforcing alternatives, lack of skill to obtain reinforcing alternatives, or increased punishment (Lewinsohn, 1974). The treatment emanating from behavior theory is referred to as behavioral activation (BA). In BA, restoring an adequate schedule of reinforcement by having the patient increase activity levels is instrumental in decreasing depressive symptoms (Lewinsohn, Biglan, \& Zeiss, 1976). BA has been reconceptualized since its original introduction. According to Martell and colleagues (Martell, Addis, \& Jacobson, 2001), BA no longer aims to replenish a broad class of positive reinforcing activities but rather to replenish positive reinforcing activities that specifically move the patient toward personal goals while blocking avoidance patterns that prevent the patient from reaching 
those goals. The approach is both functional analytic and idiographic, in which contingencies that maintain behavior for each patient are identified via self-monitoring. Avoidance patterns are changed by integrating new behaviors into a daily routine, observing the outcome, and assessing whether the behavior serves to approach or avoid personal goals.

Early treatment studies provided support for behavioral activation for depression (Brown \& Lewinsohn, 1984; Lewinsohn \& Atwood, 1969; Zeiss, Lewinsohn, \& Munoz, 1979), but as discussed by Hopko and colleagues (Hopko, Lejuez, LePage, Hopko, \& McNeil, 2003), a paradigm shift toward more cognitive explanations of psychological phenomena led to criticisms that behavioral approaches to depression were inadequate by failing to directly address depressive schemas and cognitive structures. The cognitive theory of depression, developed by Beck and colleagues (Beck et al., 1979), is based on the premise that dysfunctional cognitions and core beliefs are at the root of depression, causing both negative moods and avoidance behavior (Beck, 1970). CT seeks to change the participant's misinterpretations, self-defeating cognitions, and dysfunctional attitudes, by identifying them and recognizing the crucial link between such thoughts and the subsequent negative feelings and dysfunctional behavior that follow (Kovacs \& Beck, 1978). With the help of the cognitive therapist, the CT patient selfmonitors, challenges, and modifies depressive schemas and cognitive structures. Cognitive behavioral therapy, a merging of both cognitive and behavioral treatment modalities, resulted from this movement and has since been defined as a well-established treatment for MDD by the Task Force on the Identification and Dissemination of Efficacious Treatments (Chambless \& Ollendick, 2001).

While the clinical effectiveness of CBT has been well-documented (Chambless \& Ollendick, 2001; Dobson, 1989; Kendall, 1998; Roth \& Fonagy, 1996; Williams, 1992), whether the mechanism of action is attributable to the cognitive, behavioral, or nonspecific factors of therapy has received less attention. Some research has suggested that the greatest therapeutic gains in CBT are achieved in early sessions, when BA is administered (Hollon, Shelton, \& Davis, 1993; Otto, Pava, \& Sprich-Buckminster, 1996). In a dismantling study, Jacobson and colleagues (Jacobson et al., 1996) found that after 16 weeks of the separately identified components of CBT (i.e., behavioral activation, automatic thoughts remediation, and the full CBT regimen), all participants showed significant reductions in depressive symptoms and no significant differences in reported depression levels were observed between the three groups. The results held at 6-month, 1- and 2-year post-treatment (Gortner, Gollan, Dobson, \& Jacobson, 1998). Contrary to the investigators' a priori hypotheses, the BA condition was equally capable of producing clinically significant antidepressant effects and altering negative thinking and attributional styles compared to the cognitive therapy and "full" CBT conditions. This study called into question the assumption that dysfunctional thinking and maladaptive schemas must be directly targeted during treatment of depressed individuals (Hammen, 1997). More recently, BA has been applied to comorbid anxiety and depression (Hopko, Lejuez, \& Hopko, 2004), to psychiatric inpatients with depression (Hopko et al., 2003), and via a group modality for depressed patients (Porter et al., 2004) with promising results.

In the context of evidence-based practice, treatments that are both maximally efficacious and cost-effective have the greatest potential to impact public health. BA is a more parsimonious, efficient, and cost-effective therapy than the full CBT treatment package 
(Jacobson \& Gortner, 2000; Robinson, Wischman, \& DelVento, 1996). Behavioral approaches to depression have been characterized as easier to master by both the clinician and patient than more complex cognitive interventions (Martell et al., 2001). As such, BA might be more easily and efficiently disseminated to therapists with diverse training backgrounds (Chambless \& Hollon, 1998) and to patients with varying skill bases and cognitive functioning. The next step needed in research on BA is to determine its effectiveness when applied under conditions in which 'real world' clients present for treatment.

The present investigation sought to examine the effectiveness of BA with a sample that represents a closer approximation to patients presenting for treatment in applied settings. Unlike most depression treatment studies, participants were not excluded based on their medication status. Positive treatment outcome would not only cross validate Jacobson and colleagues' (Jacobson et al., 1996) findings with unmedicated clients, but lend support to the generalizability of BA to a medicated but still depressed patient population. Further, it would extend our recent findings of the efficacy of a behavioral activation intervention for severely depressed individuals, applied in a group modality (Porter et al., 2004),

\section{Method}

\section{$\underline{\text { Sample }}$}

Adult participants $(\mathrm{N}=25)$ seeking mental health services for MDD were recruited through public service announcements, newspaper advertisement, solicitations from community professionals, and other healthcare agencies. All participants met DSM-IV criteria for MDD based on the Structured Clinical Interview for Diagnosis of DSM-IV-Non Patient (SCID-NP) (First, Spitzer, Gibbon, \& Williams, 1997). Participants scored at least 20 on the Beck Depression Inventory-II (BDI-II) (Beck, Steer, \& Brown, 1996), and 14 or greater on the Revised-Hamilton Rating Scale for Depression (R-HRSD) (Warren, 1996).

Exclusion criteria included current bipolar $(n=2)$ or psychotic disorders $(n=1)$, panic disorder $(n=0)$, current alcohol or other substance abuse $(n=0)$, organic brain syndrome $(n=0)$, obsessive compulsive disorder $(\mathrm{n}=0)$ and mental retardation $(\mathrm{n}=0)$. Suicide risk was regularly evaluated via responses to the suicide items on the BDI-II, the R-HRSD, and at the start of the investigation by the SCID-NP, as well as throughout the study by monitoring any verbalizations of suicidal ideations made by the patient.

Setting, Assessors and Therapists

All assessment and treatment sessions were conducted in the outpatient service of a psychology training clinic that was associated with an APA-approved clinical psychology doctoral program. Doctoral graduate students in clinical or counseling psychology conducted all assessment interviews. Therapists had previous training in the cognitive-behavioral treatment of depression, and for purposes of the present investigation, received an additional 12 hours of training in the use of BA therapy. 


\section{$\underline{\text { Experimental Design }}$}

A pre-test post-test wait list control group design was used. As this study was an early attempt at cross-validating Individual BA this type of control arrangement is appropriate instead of a comparative treatment design which assumes prior evidence of efficacy of two or more treatments. Additionally Human Subjects concerns mitigated use of a purely "no treatment" control group design. All participants were randomly assigned to either the immediate treatment (IT) or waitlist condition (WL). All participants were evaluated before treatment, at the termination of treatment, and at 3 months follow-up on principal dependent measures. Waitlist participants were additionally assessed before receiving treatment after the post wait period. Depressive symptomatology was also assessed bi-weekly using the BDI-II during the waitlist phase and weekly during the treatment phase.

\section{$\underline{\text { Measures }}$}

Depressive symptomatology was measured in three ways. First, the SCID-NP (First et al., 1997) was administered during the screening and post-test visit to assess if diagnostic criteria were met for major depressive disorder at pretest, posttest and 3-month follow-up. The SCID-NP is a broad-based structured clinical interview that covers 50 major DSM-IV disorders. Reliability was established by comparing the independent evaluation by two or more raters across a group of participants (Spitzer, Williams, Gibbon, \& First, 1992). The BDI-II (Beck et al., 1996) was used to assess depressive symptomatology at screening, every second week during the 6-week waitlist period, at every treatment session, and at 3 months follow-up. The R-HRSD (Warren, 1996) was also used to assess depressive symptomatology at pretest, posttest, and 3-month follow-up.

\section{$\underline{\text { Treatment Integrity }}$}

Protocol outlines were supplied to each therapist for each session after initial training. The treatment fidelity measure used in this study was a modified version of the National Institute of Mental Health Collaborative Study Psychotherapy Rating Scale (CSPRS) (Hollon, Evans, Elkin, \& Lowery, 1984). The CSPRS included the procedural steps in BA, along with a checklist of prescribed BA techniques. Also included was a list of proscribed cognitive therapy techniques. Trained observers then viewed a random sample of the video taped treatment sessions (11\%), checking off the presence of each step in the outline, along with the specific treatment interventions used in that session. Inter-rater reliability checks were then performed on this same sample of videotapes and was found to be $88 \%$, indicating that the raters agreed $88 \%$ of the time that therapists were delivering BA according to the protocol described in the treatment manual. Weekly research team meetings with a licensed clinical psychologist (the second author) were conducted to discuss cases and reduce therapist drift.

\section{$\underline{\text { Procedure }}$}

Adult participants seeking mental health services for major depression were recruited for this study via advertisements, public service announcements, public postings, and solicitations 
from community professionals and health care agencies. Interested individuals phoned and underwent an initial telephone screening. During this screening, the nature of the study was explained and potential participants were assessed for depressive symptomatology. Potential participants were also asked if they were currently using any psychotropic medication or in psychotherapy. Those who were not experiencing symptoms of depression, who had been taking prescription medication for their depression for less than six weeks, and/or who were currently in other psychological treatments were deemed ineligible for participation. Appropriate referrals for mental health services were offered to all ineligible callers.

Eligible individuals were scheduled to participate in an in-person screening interview. During the screening interview, participants provided informed consent and were assessed for eligibility. Eligible participants $(n=25)$ were randomly assigned to either WL or IT conditions. Participants in the IT condition were scheduled for their initial treatment appointment. WL participants were told they would first participate in an "assessment phase" for which information about their depression would be gathered in advance to starting therapy. WL participants then visited the clinic every other week, for a total of three visits, while on the 6week waitlist.

\section{$\underline{\text { Treatment Protocol }}$}

BA treatment utilized in the present investigation followed closely the model used in the Jacobson et al. (Jacobson et al., 1996) investigation. Thus the purpose of BA is to activate participants so that they can break a passive approach to life and maximize their opportunity to make contact with natural, positive reinforcers in their environment (Martell et al., 2001). The emphasis of BA is on "focused activation," as opposed to simple activity at random. This includes not only finding behaviors and activities that will be positively reinforcing, but paying close attention to the activities with which one is participating (e.g., noticing colors, noises, and smells associated with the activity). This attention to the experience is very similar to the mindfulness training found in Morita therapy (LeVine, 1998) and dialectical behavior therapy (Jacobson, Martell, \& Dimidjian, 2001; Linehan, 1993).

The goals of BA are to (1) determine the life circumstances that precipitated the depression, (2) determine the coping patterns that maintained and exacerbated the depression (e.g., chronic negativity, social withdrawal), and (3) develop a treatment plan for improving the coping patterns and provide access to more reinforcing life circumstances (Martell et al., 2001). The therapy is delivered in a directive manner, but the participant and therapist choose the direction in concert. The therapist coaches the participant to learn a core set of BA skills, but because the skill form varies from participant to participant, the BA therapist is required to be flexible, proficient, and able to coach a wide range of unique participants.

Each BA treatment session involves a distinctive beginning, middle, and end. The beginning of each session includes greeting the participant and administering the BDI-II. Issues to be covered throughout the rest of the session are then placed on an agenda as the therapist and participant work collaboratively to determine the most important topics for that week. Next, the completed BDI-II is reviewed, paying close attention to the specific questions that target suicidal 
behavior and weekly activity levels. Any homework assignments the participant completed between sessions are also discussed.

The middle segment of the session involves working jointly on the previously set agenda items. The therapist typically does not stray from the prescribed agenda unless an emergent issue arises (e.g., suicidality). Reviewing self-monitoring records, identifying avoidance patterns, and brainstorming behavioral alternatives are activities that often occur in this part of session. As the session closes, the therapist briefly reviews topics covered and assigns homework. As treatment progresses, the participant begins to assume responsibility for reviewing the session and assigning homework to him or herself at the end of sessions.

\section{End of Treatment and Follow-Up Assessment}

One week following the end of treatment, each participant returned to the clinic for posttest assessment. Each participant met with a clinical assessor who administered the BDI-II and the SCID-NP. Finally, the assessor independently completed the R-HRSD. The same routine was completed at the 3-month follow-up session.

\section{$\underline{\text { Analytic Plan }}$}

The analytic plan involved two approaches. The first was a 'completer' approach including only those participants $(n=17)$ who attended a minimum of six sessions of BA. The second model was an intention to treat (ITT) approach, including all randomized participants $(n=25)$. Finally, analyses of the clinical significance of outcome were conducted.

Completer approach. To determine the degree to which depressive symptoms were affected by treatment in each group, separate repeated measures analyses of variances (ANOVAs) were conducted on BDI-II and R-HSRD scores for each group with time entered as the within subjects factor (pre-treatment, post-treatment, 3-month follow-up). To evaluate change due to the time passage only, using a mixed model ANOVA, BDI-II scores of WL participants during the WL period were compared to BDI-II scores of IT participants during the same period of time. Group was entered as a between-subjects factor and time (week 1, 3, and 5) was entered as the within-subjects factor.

Intention to treat approach. ITT analyses, where all randomized cases are included in analyses, are required in standardized reporting guidelines. (Moher, Schulz, \& Altman, 2001) In the ITT approach, the same analyses above were performed, but including all 22 randomized subjects. To include drop-outs, the last available BDI-II or R-HSRD score was used as the score for all time points following their last visit (i.e., last value carried forward). The intent to treat sample was also used to explore whether participants varied in their responses to the intervention depending on their medication status. Two repeated measures ANOVAs were conducted with Medication Status as a between-subjects independent variable and Time as a within-subjects independent variable. R-HSRD and BDI-II were the dependent variables in the models.

Clinical Significance Testing. In recent years a movement in the psychotherapy literature has occurred towards the addition of clinical significance tests to more traditional null hypothesis 
significance tests (e.g., Jacobson, Follette, \& Revenstorf, 1984; Jacobson, Roberts, Berns, \& McGlinchey, 1999; Jacobson \& Truax, 1991; Kendall, Marrs-Garcia, Nath, \& Sheldrick, 1999). The purpose of clinical significance tests is to differentiate treatment effects that are meaningful in the real world from those that merely have a low $p$-value. Consequently, we also conducted analyses of clinical significance. As recommended by Jacobson et al. (1999), we compared endof-treatment means to published norms for both depressed and non-depressed samples.

\section{$\underline{\text { Participant Characteristics }}$}

Demographic information for the entire sample is displayed in Table 1. Of the 25 participants, 32\% were female. Participants were largely Caucasian (88\%) and on average 38 years of age. Almost one-third of participants (32\%) reported taking psychotropic medication to treat their depression. Four participants reported taking fluoxetine (Prozac), two were being treated with citalopram hydrobromide (Celexa), one reported taking paroxetine hydrochloride (Paxil), and another did not disclose the antidepressant medication he/she was taking. All medicated participants must have been taking the drug for a period of at least 6 weeks to be included in the study. No participants reported current participation in other psychotherapy.

Table 1: Baseline Characteristics of Wait List, Immediate Treatment and Total Sample

\begin{tabular}{|c|c|c|c|}
\hline Variable & $\begin{array}{c}\text { WL } \\
(n=12)\end{array}$ & $\begin{array}{c}\mathrm{IT} \\
(\mathrm{n}=13) \\
\end{array}$ & $\begin{array}{l}\text { Total Sample } \\
\qquad(\mathrm{N}=25)\end{array}$ \\
\hline \multicolumn{4}{|l|}{ Gender } \\
\hline Female & $33 \%$ & $31 \%$ & $32 \%$ \\
\hline Age & $41.75(13.05)$ & $35.46(12.07)$ & $38.48(12.69)$ \\
\hline \multicolumn{4}{|l|}{ Ethnicity } \\
\hline Caucasian & $92 \%$ & $85 \%$ & $88 \%$ \\
\hline African American & $8 \%$ & $0 \%$ & $4 \%$ \\
\hline Hispanic & $0 \%$ & $8 \%$ & $4 \%$ \\
\hline Asian & $0 \%$ & $7 \%$ & $4 \%$ \\
\hline Medication Status & $25 \%$ & $39 \%$ & $32 \%$ \\
\hline Baseline BDI-II & $29.91(5.71)$ & $31.92(6.14)$ & $30.96(5.90)$ \\
\hline Baseline R-HSRD & $19.90(3.44)$ & $18.07(3.37)$ & $18.96(3.46)$ \\
\hline Completer & $67 \%$ & $69 \%$ & $68 \%$ \\
\hline
\end{tabular}


Participants who dropped out did not differ from those who completed the study in terms of gender, ethnicity, age, education, income, whether they had been in psychotherapy in the past, whether they were currently receiving antidepressant pharmacotherapy, or whether they had ever received antidepressant pharmacotherapy.

Of the 17 completers, 13 participants entered the study with an Axis I diagnosis of MDD, Recurrent/Moderate, while 2 participants were diagnosed with MDD, Recurrent/Severe without psychotic features (see Table 2). One participant suffered from MDD, Recurrent/Mild and one from MDD, Single Episode/Mild. The SCID-NP identified 3 participants with dysthymic disorder, yielding a formal diagnosis of "double depression." No participants obtained a formal Axis II diagnosis. Finally, Axis V Global Assessment of Functioning (GAF) scores ranged from 51-68, with a mean GAF of 60.

\section{$\underline{\text { Completer Analyses }}$}

No significant differences between IT and WL participants were observed at time of initial intake on either the BDI-II, $t(15)=1.04, p=\mathrm{ns}$, or the RHRSD, $t(15)=.94, p=\mathrm{ns}$. IT and WL participants started treatment at comparable levels of symptom severity. The repeated measures ANOVA for the BDI revealed a significant reduction in symptoms from pre to post testing, $\mathrm{F}(2,14)=15.82, \mathrm{p}<.01$. There was no significant group by time interaction. The repeated measures ANOVA for the R-HSRD also revealed significant symptom reductions from pre to post treatment, $\mathrm{F}(2,14)=9.47, \mathrm{p}<.01$, and likewise no significant group by time interaction. On both dependent measures, depression scores declined over time (see Figures 1 and 2). In light of the stability of these measures for Waitlist participants from the pretest to post wait period, these data suggest that symptom reductions were attributable to the intervention and not due to time alone.

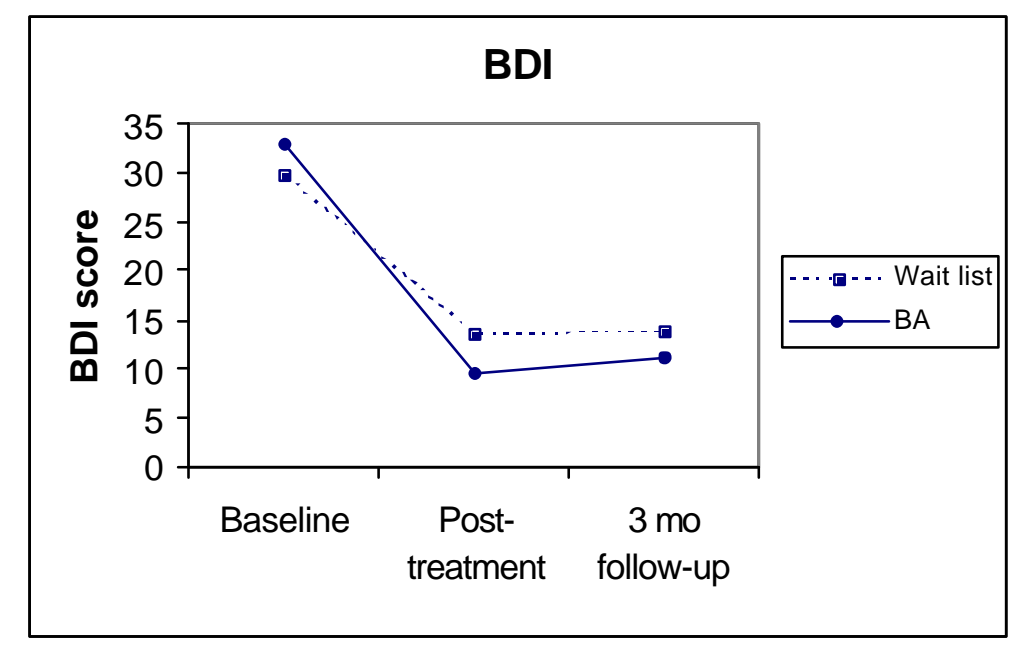

Figure 1. Mean Beck Depression Inventory (BDI) scores at baseline, post-treatment and 3month follow-up. 


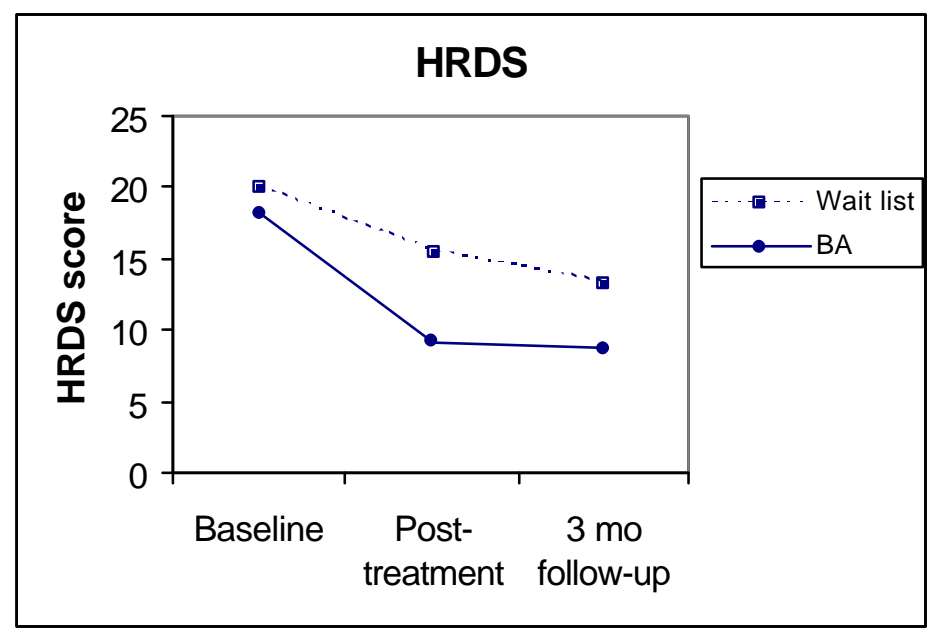

Figure 2. Mean Hamilton Rating Depression Scale scores at baseline, post-treatment and 3month follow-up.

\section{$\underline{\text { Intent to Treat Analysis }}$}

Intent to treat analyses were consistent with completer analyses. Both repeated measures ANOVA models for BDI, $\mathrm{F}(2,22)=27.89, \underline{\mathrm{p}}<.01$, and $\mathrm{HRSD}, \mathrm{F}(2,22)=6.96, \underline{\mathrm{p}}<.01$, revealed significant symptom reductions, but no significant interactions of time and group. Like the completer analyses, these analyses showed that depression scores declined significantly from pretest to posttest.

Additional post-hoc analyses were conducted to determine whether the changes in BDI scores could be a function of time rather than a function of treatment. As already indicated scores for subjects in the Immediate and Waitlist conditions were comparable at the pretesting. Additional comparisons of WL and IT at 6 weeks indicated that the IT participants showed significant changes BDI scores compared to WL participants. Results indicated that IT participants' BDI scores at week 6 were already significantly lower than those of WL participants at week $1[\mathrm{~F}(1,20)=4.26, \mathrm{p}=.05]$. Thus, reductions in BDI scores reported by IT participants at week 6 appeared to be a function of treatment rather than time. These data, coupled with the significant reduction in symptoms noted when WL participants were subsequently assigned to treatment, clearly indicates the symptom reductions were due to Behavioral Activation intervention.

BDI Classification. Beck, Steer, and Brown (Beck et al., 1996) recommended the following classification scheme for BDI scores: 0 - 13, minimal depression; $14-19$, mild depression; $20-28$, moderate depression; 29 - 63, severe depression. According to this scheme, at week 1 of treatment, 11 participants in the current sample were classified as severely depressed, 6 as moderately depressed, 5 as mildly depressed, and 0 as minimally depressed. At week 10, 3 participants were classified as severely depressed, 5 as moderately depressed, 1 as mildly depressed, and 13 as minimally depressed. 
Effect of Medication Status. Exploratory analyses were conducted to determine whether participants on medication improved to a greater extent than those who were unmedicated. For BDI-II, a significant main effect of Time was revealed, $F(2,22)=24.27, p<.001$, but no significant Time by Medication Status interaction, $F(2,22)=.98, \underline{p}=.39$. Results for the RHSRD were similar. A significant main effect of Time, $F(2,22)=6.02, \mathrm{p}<.01$ and no significant Time by Medication Status interaction, $\mathrm{F}(2,22)=.02, \mathrm{p}=.97$. Therefore, it can be concluded that there was no evidence for a differential response to the intervention by medication status.

\section{Clinical Significance}

As recommended by Jacobson et al. (1999), we compared post-treatment BDI scores to norms for both depressed and non-depressed samples. Across treatment groups, at the first treatment visit, the current sample reported a mean BDI of $27.73(\underline{\mathrm{sd}}=8.29)$. This was not significantly different from the mean of $28.64(\underline{\mathrm{d} d}=11.75)$ that Steer, Ball, Ranieri, and Beck (1999) reported among a group of 210 depressed outpatients [t $(230)=0.47, \underline{p}=n s]$. It was significantly different from the mean of $12.55(\underline{\mathrm{sd}}=9.93)$ among non-depressed undergraduates reported by Beck et al. $(1996)[\underline{\mathrm{t}}(140)=7.64, \underline{\mathrm{p}}<.01]$ and the $11.86(\underline{\mathrm{sd}}=8.06)$ reported by Steer and Clark (1997) [t $(180)=8.45, \underline{\mathrm{p}}<.01]$.

Across groups, mean post-treatment BDI in the present sample was $14.00(\underline{\mathrm{sd}}=10.66)$. This was not significantly different from the norms for non-depressed undergraduates reported by Beck et al (1996) [ $\underline{\mathrm{t}}(140)=0.59, \underline{\mathrm{p}}=\mathrm{ns}]$ and by Steer and Clark $(1997)[\underline{\mathrm{t}}=0.91, \underline{\mathrm{p}}=\mathrm{ns}]$. However, it was significantly different from the norms for depressed outpatients reported by Steer et al. (1999) [ $\mathrm{t}(230)=6.07, \mathrm{p}<.01]$. In sum, prior to treatment, the current sample was not statistically different from a sample of depressed outpatients, but was significantly more depressed than those reported by two groups of non-depressed undergraduates. Following treatment the converse pattern was evident. The current sample reported significantly lower BDI scores than the same sample of depressed outpatients and were not significantly different from either of the two samples of non-depressed undergraduates. These data indicate that the present sample met criteria for "caseness" before treatment and showed clinical significant improvement following treatment. Earlier analyses further indicated this improvement to be attributable to treatment rather than the passage of time.

\section{$\underline{\text { Treatment Fidelity }}$}

On $100 \%$ of the observed occasions, raters responded "yes" to the checklist item, "therapist implemented behavioral activation interventions." Furthermore, on $100 \%$ of the observed occasions, raters responded "no" to the item, "therapist did use cognitive interventions," indicating cognitive therapy was not implemented at any point throughout treatment in the observed sessions. Rater data revealed that therapists did not use cognitive interventions during BA treatment. 


\section{Discussion}

The purpose of this study was to examine the effectiveness of BA as a treatment for MDD in a sample of medicated and unmedicated participants. Results revealed that BA led to significantly reduced self-ratings of depression, independent assessor ratings of depression, and formal diagnoses of MDD from pretesting to 3-month follow-up. Additionally, neither the use of psychotropic medications nor participants' status as IM or WL explained the findings. The endstate functioning of participants in this study achieved clinically significant results in 6 - 10 treatment sessions. The full CBT treatment regimen typically includes approximately 20 treatment sessions.

Results are consistent with previous studies (e.g., Hopko et al., 2004; Hopko et al., 2003; Jacobson et al., 1996; Porter et al., 2004) that have demonstrated efficacy of BA among depressed patients. The results extend Jacobson et al's (1996) original demonstration of the efficacy of BA by supporting the efficacy of BA in a sample that was heterogenous in terms of medication status. Because patients seeking psychotherapy for depression are often medicated prior to therapy, it is important to determine whether behavioral treatments produce a benefit for this population. Our previous investigation (Porter et al., 2004) demonstrated efficacy of groupadministered BA in medicated and unmedicated patients. The current investigation builds on the evidence of the efficacy of BA in both medicated (but still depressed) and unmedicated patients.

As in all research, this investigation has some limitations. The dropout rate was $32 \%$, not unlike dropout rates seen in psychiatric community outpatient clinics, which typically range from 20-60\% (Simons, Levine, Lustman, \& Murphy, 1984), and better than other effectiveness studies that report dropout rates between 40-60\% (Chambless \& Ollendick, 2001).

Continued research with larger sample sizes is needed to further validate the effectiveness and generalizability of BA in real world settings and with patient samples with fewer exclusionary criteria. One direction for future BA research may include broadening the range of patients and settings in which BA appears to be effective. For instance, a next step might include testing the effectiveness of BA in other "real-world" and naturalistic settings (e.g., private and public mental health practice, instead of an academic clinical research setting), with still less stringent inclusion criteria (e.g., include those exhibiting suicidal behaviors and/or other comorbid Axis I and II disorders). This would provide an increment of "real world-ness" beyond the addition of medicated clients in the present study. Another future investigation might entail examination of the limitations of Individual BA with more severe patient samples, i.e. those with co-morbid conditions and severity levels. The severity and chronic nature of this disorder have widespread community implications, as both have been correlated with a higher financial burden to the community (Lecrubier, 2001). Finally, Individual BA should be compared to another efficacious treatment for MDD, i.e. CBT, medications or Interpersonal Therapy.

The results of this investigation affirm that BA shows great promise as an effective treatment strategy for both medicated and unmedicated individuals suffering from MDD. This study supports the notion of BA as a sufficient and cost-effective therapeutic tool. As in CBT, however, finding both statistically and clinically significant improvements in depression via BA does not imply that all components of BA are necessary to achieve that improvement or that it is 
equally efficacious will all patient samples. Future research that aims to uncover the specific mechanisms of action of BA may further our understanding of the process of change and how to develop a still more parsimonious treatment.

\section{References}

Angst, J. (1998). The prevalence of depression. In M. Briley \& S. Montgomery (Eds.), Antidepressant therapy in the dawn of the third millennium (1st ed., pp. 191-212). St Louis: Mosby.

Beck, A. T. (1970). Cognitive therapy: Nature and relation to behavior therapy. Behavior Therapy, 1, 184-200.

Beck, A. T., Rush, A. J., Shaw, B. F., \& Emery, G. (1979). Cognitive therapy of depression. New York: Guilford Press.

Beck, A. T., Steer, R. A., \& Brown, G. K. (1996). Manual for the Beck Depression Inventory-II. San Antonio, TX: The Psychological Corporation.

Brown, R. A., \& Lewinsohn, P. M. (1984). A psychoeducational approach to the treatment of depression: Comparison of group, individual, and minimal contact procedures. Journal of Consulting and Clinical Psychology, 52, 774-783.

Chambless, D. L., \& Hollon, S. D. (1998). Defining empirically supported therapies. Journal of Consulting and Clinical Psychology, 66, 7-18.

Chambless, D. L., \& Ollendick, T. H. (2001). Empirically supported psychological interventions: Controversies and evidence. Annual Review of Psychology, 52, 685-716.

Dobson, K. S. (1989). A meta-analysis of the efficacy of cognitive therapy for depression. Journal of Consulting and Clinical Psychology, 57, 414-419.

First, M. B., Spitzer, R. L., Gibbon, M., \& Williams, J. B. W. (1997). Structured clinical interview for DSM-IV axis I disorders-clinical version (SCID-CV). Washington, DC: American Psychiatric Press.

Gortner, E. T., Gollan, J. K., Dobson, K. S., \& Jacobson, N. S. (1998). Cognitive-behavioral treatment for depression: Relapse prevention. Journal of Consulting and Clinical Psychology, 66, 377-384.

Hammen, C. (1997). Depression. East Sussex, UK: Psychology Press Ltd.

Hollon, S. D., Evans, M. D., Elkin, I., \& Lowery, A. (1984). System for rating therapies for depression. Paper presented at the 92nd Annual Convention of the American Psychological Association, Toronto, Ontario, Canada. 
Hollon, S. D., Shelton, R. C., \& Davis, D. D. (1993). Cognitive therapy for depression: Conceptual issues and clinical efficacy. Journal of Consulting and Clinical Psychology, $61,270-275$.

Hopko, D. R., Lejuez, C. W., \& Hopko, S. D. (2004). Behavioral activation as an intervention for coexistent depressive and anxiety symptoms. Clinical Case Studies, 3, 37-48.

Hopko, D. R., Lejuez, C. W., LePage, J. P., Hopko, S. D., \& McNeil, D. W. (2003). A brief behavioral activation treatment for depression. A randomized pilot trial within an inpatient psychiatric hospital. Behavior Modification, 27, 458-469.

Jacobson, N. S., Dobson, K. S., Truax, P. A., Addis, M. E., Koerner, K., Gollan, J. K., et al. (1996). A component analysis of cognitive-behavioral treatment for depression. Journal of Consulting and Clinical Psychology, 64, 295-304.

Jacobson, N. S., Follette, W. C., \& Revenstorf, D. (1984). Toward a standard definition of clinically significant change. Behavior Therapy, 17, 308-311.

Jacobson, N. S., \& Gortner, E. T. (2000). Can depression be de-medicalized in the 21st century: Scientific revolutions, counter-revolutions and the magnetic field of normal science. Behaviour Research \& Therapy, 38, 103-117.

Jacobson, N. S., Martell, C. R., \& Dimidjian, S. (2001). Behavioral activation treatment for depression: Returning to contextual roots. Clinical Psychology: Science and Practice, 8, 255-270.

Jacobson, N. S., Roberts, L. J., Berns, S. B., \& McGlinchey, J. B. (1999). Methods for defining and determining the clinical significance of treatment effects: Description, application, and alternatives. Journal of Consulting and Clinical Psychology, 67, 300-307.

Jacobson, N. S., \& Truax, P. (1991). Clinical significance: A statistical approach to defining meaningful change in psychotherapy research. Journal of Consulting and Clinical Psychology, 59, 12-19.

Kendall, P. C. (1998). Empirically supported psychological therapies. Journal of Consulting and Clinical Psychology, 66, 3-6.

Kendall, P. C., Marrs-Garcia, A., Nath, S. R., \& Sheldrick, R. C. (1999). Normative comparisons for the evaluation of clinical significance. Journal of Consulting and Clinical Psychology, 67, 285-299.

Kessler, R. C., Berglund, P., Demler, O., Jin, R., Koretz, D., Merikangas, K. R., et al. (2003). The epidemiology of major depressive disorder: Results from the National Comorbidity Survey Replication (NCS-R). Journal of the American Medical Association, 289, 30953105. 
Kimerling, R., Ouimette, P. C., Cronkite, R. C., \& Moos, R. H. (1999). Depression and outpatient medical utilization: a naturalistic 10-year follow-up. Annals of Behavioral Medicine, 21, 317-321.

Kovacs, M., \& Beck, A. T. (1978). Maladaptive cognitive structures in depression. American Journal of Psychiatry, 135, 525-533.

Lecrubier, Y. (2001). The burden of depression and anxiety in general medicine. Journal of Clinical Psychiatry, 62, 4-9; discussion 10-11.

LeVine, P. (1998). Morita therapy and the true nature of anxiety-based disorders (Shinkeishitsu). Albany, NY: State University of New York Press.

Lewinsohn, P. M. (1974). A behavioral approach to depression. In R. M. Friedman \& M. M. Katz (Eds.), The psychology of depression: Contemporary theory and research. New York: Wiley.

Lewinsohn, P. M., \& Atwood, G. E. (1969). Depression: A clinical-research approach. Psychotherapy: Theory, Research, and Practice, 6, 166-171.

Lewinsohn, P. M., Biglan, A., \& Zeiss, A. S. (1976). Behavioral treatment of depression. In P. O. Davidson (Ed.), The behavioral management of anxiety, depression and pain (pp. 91146). New York: Brunner/Mazel.

Linehan, M. M. (1993). Cognitive-behavioral treatment of borderline personality disorder. New York: Guilford.

Martell, C. R., Addis, M. E., \& Jacobson, N. S. (Eds.). (2001). Depression in context. Strategies for guided action. New York: W. W. Norton.

McLean, P. D., Ogston, K., \& Grauer, L. (1973). A behavioral approach to the treatment of depression. Journal of Behavior Therapy and Experimental Psychiatry, 4.

Moher, D., Schulz, K. F., \& Altman, D. (2001). The CONSORT statement: Revised recommendations for improving the quality of reports of parallel-group randomized trials. Journal of the American Medical Association, 285, 1987-1991.

NIMH (2005). Breaking Ground, Breaking Through: The Strategic Plan for Mood Disorders Research. National Institutes of Mental Health, U. S. Department of Health and Human Services.

Otto, M. W., Pava, J. A., \& Sprich-Buckminster, S. (1996). Treatment of major depression: Application and efficacy of cognitive-behavioral therapy. In M. H. Pollack \& M. W. Otto (Eds.), Challenges in clinical practice: Pharmacologic and psychosocial strategies (pp. 31-52). New York: Guilford Press. 
Persons, J. B., Burns, D. D., \& Perloff, J. M. (1988). Predictors of dropout and outcome in cognitive therapy for depression in a private practice setting. Cognitive Therapy and Research, 12, 557-575.

Porter, J. F., Spates, R. C., \& Smitham, S. (2004). Behavioral activation group therapy in public mental health settings: A pilot investigation. Professional Psychology: Research and Practice, 35, 297-301.

Robinson, P., Wischman, C., \& DelVento, A. (1996). Treating depression in primary care. Reno, NV: Context Press.

Roth, A. D., \& Fonagy, P. (1996). What works for whom? A critical review of psychotherapy research. New York: Guilford Press.

Simons, A. D., Levine, J. L., Lustman, P. J., \& Murphy, G. E. (1984). Patient attrition in a comparative outcome study of depression. A follow-up report. Journal of Affective Disorders, 6, 163-173.

Spitzer, R. L., Williams, J. B., Gibbon, M., \& First, M. B. (1992). The Structured Clinical Interview for DSM-III-R (SCID). I: History, rationale, and description. Archives of General Psychiatry, 49, 624-629.

Steer, R. A., Ball, R., Ranieri, W. F., \& Beck, A. T. (1999). Dimensions of the Beck Depression Inventory-II in clinically depressed outpatients. Journal of Clinical Psychology, 55, 117128.

Steer, R. A., \& Clark, D. A. (1997). Psychometric characteristics of the Beck Depression Inventory-II with college students. Measurement \& Evaluation in Counseling \& Development, 30, 128-137.

Steinbrueck, S. M., Maxwell, S. E., \& Howard, G. S. (1983). A meta-analysis of psychotherapy and drug therapy in the treatment of unipolar depression with adults. Journal of Consulting and Clinical Psychology, 51, 856-863.

Warren, W. L. (1996). Manual for the revised Hamilton Rating Scale for depression: Western Psychological Services.

Williams, J. M. G. (1992). The psychological treatment of depression. A guide to the theory and practice of cognitive behaviour therapy (2nd ed.). London: Routledge. 
World Health Organization. (2005). WHO initiative on depression and public health. Accessed January 1, 2005, 2005, from http://www.who.int/mentalhealth/management/depression/definition/en/print.html

Zeiss, A. M., Lewinsohn, P. M., \& Munoz, R. F. (1979). Nonspecific improvement effects in depression using interpersonal skills training, pleasant activity schedules, or cognitive training. Journal of Consulting and Clinical Psychology, 47, 427-439.

\section{Author contact information:}

Jenifer M. Cullen

Arbour-Fuller Hospital/Advocates Inc.

200 May Street

S. Attleboro, MA 02703

508-838-2282

jenifer_cullen@hotmail.com

C. Richard Spates

Western Michigan Univesity

Department of Clinical Psychology

Kalamazoo, MI 49008

269-387-4329

rspates@wmich.edu

Sherry L. Pagoto

Department of Medicine

Division of Preventive and Behavioral Medicine

University of Massachusetts Medical School

55 Lake Avenue North

Worcester, MA 01655

508-856-2092

sherry.pagoto@umassmed.edu

Neal Doran

University of California, San Diego

Dept. of Psychiatry/SDVAMC

3350 La Jolla Village Dr.

San Diego, CA 92161

858-552-8585 ext 5531

nmdoran@ucsd.edu 